\title{
Comparison of modified biophysical profile and Doppler ultrasound in prediction of perinatal outcome in high-risk pregnancies
}

\author{
Khushboo Malhotra*, Archana Kumari, H. P. Anand
}

Department of Obstetrics and Gynecology, VMMC and Safdarjung Hospital, New Delhi, India

Received: 16 April 2020

Revised: 22 May 2020

Accepted: 28 May 2020

\section{*Correspondence:}

Dr. Khushboo Malhotra,

E-mail: khushi_malh@yahoo.co.in

Copyright: (c) the author(s), publisher and licensee Medip Academy. This is an open-access article distributed under the terms of the Creative Commons Attribution Non-Commercial License, which permits unrestricted non-commercial use, distribution, and reproduction in any medium, provided the original work is properly cited.

\section{ABSTRACT}

Background: The objective of this present study was to compare MBPP and umbilical artery Doppler flow in highrisk pregnant women in prediction of perinatal outcome.

Methods: A cohort study was done on 150 high-risk pregnant women over 16 months. Antenatal women with singleton pregnancy who delivered within 48 hours of performing MBPP and Doppler USG, with presence of $\geq 1$ high-risk factor like pre-eclampsia/gestational HTN, BOH, post-dated pregnancy, FGR, GDM, maternal heart disease, anaemia, hypothyroidism and IHCP were included in the study. MBPP (NST and AFI) and umbilical artery Doppler was performed. Perinatal outcome was measured in terms of stillbirth/IUD, LBW, Apgar $<7$ at 5 minutes, admission to NICU, neonatal death within 48 hours of delivery, MSL and neonatal seizures within 24-48 hours. Quantitative variables were compared using independent t-test/Mann Whitney test. Qualitative variables were correlated using Chi square test/Fisher exact test. Sensitivity, specificity, NPV, PPV were calculated and p-value $<0.05$ was considered statistically significant. Data analysis was done using social sciences (SPSS) licensed version 21.0.

Results: Majority belonged to the age group 21-25 years and were between 37-40 weeks of gestation. It was found that highest perinatal complications occurred in those with both abnormal MBPP and Doppler followed by those with only abnormal MBPP (p-value<0.0001).

Conclusions: MBPP is a better predictor of perinatal outcome compared to umbilical artery Doppler USG in highrisk pregnant women. MBPP should be done in all high-risk pregnancies even if Doppler is normal. Both the tests must be performed in all high-risk pregnancies to improve perinatal outcome.

Keywords: High risk pregnancy, Modified biophysical profile, Non-stress test, Perinatal outcome, Umbilical artery doppler

\section{INTRODUCTION}

Perinatal mortality is one of the most important public health issues in the developing countries and high-risk pregnancy is a major contributor of increased perinatal morbidity and mortality. ${ }^{1}$ About 7.3 million perinatal deaths occur every year around the world and majority occur in Asia. In India alone, around 890000 deaths of the infants occur annually. ${ }^{2}$ Antepartum foetal surveillance is of immense importance for detection of foetal compromise in utero in high risk pregnancies.
Various tests that assess high risk pregnancy are nonstress test (NST), contraction stress test (CST), biophysical profile (BPP), modified BPP (MBPP) and Doppler velocimetry. Various authors compared the efficiency of NST, BPP and abnormal Doppler findings in predicting adverse perinatal outcome in high risk pregnancies in search of a better tool for perinatal outcome. $^{3}$

NST is a primary foetal surveillance tool. It is simple, non-invasive, and inexpensive; and has no 
contraindications. NST utilizes the observation that the occurrence of accelerations of the foetal heart rate in response to foetal movements is a reliable indicator of immediate foetal wellbeing. However, an abnormal NST is nonspecific and needs further testing. ${ }^{4}$

The modified biophysical profile (MBPP) suggested by Nageotte et al, combines non-stress test (NST) as a short term marker of foetal status and the amniotic fluid index (AFI) as marker of long term placental function and is easier to perform and less time consuming than complete biophysical profile or contraction stress test. ${ }^{5}$ Also, MBPP is considered to be as effective as complete biophysical profile.

Doppler ultrasound is a non- invasive procedure that aims to evaluate blood flow in the vessels supplying the placenta and the foetus. Different vessels examined are uterine artery, umbilical artery, middle cerebral artery and ductus venosus. It is necessary in pregnancy complicated by FGR, oligohydramnios, twin-twin transfusion syndrome and discordant twins.

High-risk pregnancies increase the maternal and foetal morbidity and mortality; and there is a need for appropriate investigation which can diagnose it early and improve perinatal outcome. Hence, this study was undertaken to compare MBPP (NST and amniotic fluid index) and umbilical artery Doppler findings in assessing the perinatal outcome in high-risk pregnancy.

The objective of this study was to compare MBPP and umbilical artery Doppler flow in high-risk pregnant women for prediction of perinatal outcome.

\section{METHODS}

This was a cohort study conducted in the department of obstetrics and gynecology, in a tertiary care centre of North India over a period of 16 months. 150 cases of high-risk antenatal women with singleton pregnancy, who delivered within 48 hours of performing MBPP and Doppler ultrasound and who were willing to participate in the study were enrolled into the study after informed consent and ethical clearance. High-risk pregnancy included any of the following - preeclampsia or gestational hypertension, bad obstetric history, post dated pregnancy (>40 weeks), foetal growth restriction (FGR). gestational diabetes mellitus (GDM), maternal heart disease, anaemia, intrahepatic cholestasis of pregnancy (IHCP) and hypothyroidism. In all cases, accurate gestational age was established from detailed menstrual history and first trimester ultrasound. Detailed history, examination, investigation and monitoring were done as per the hospital protocol. These women were subjected to umbilical artery Doppler study and modified BPP evaluation as close to delivery as possible. Results of these tests were correlated with perinatal outcome. Termination of pregnancy was done as per the routine management protocol. Maternal outcome in terms of type delivery (spontaneous/induced) and mode of delivery were recorded.

Doppler study was considered abnormal when any of the following parameters were met:

- Pulsatility index of $\mathrm{UA}>95^{\text {th }}$ percentile for the gestational age.

- Absence or reversal of end diastolic flow in umbilical artery or persistent early diastolic notch in uterine artery.

- $\quad$ S/D ratio more than 3 in umbilical artery after 30 weeks of gestation and more than 2.6 in uterine artery was considered abnormal.

NST was considered as reactive with more than or equal to 2 accelerations of more than or equal to 15 beats/minute lasting for more than or equal to 15 seconds, with good beat-to-beat variability and no decelerations. AFI less than or equal to 5 and more than or equal to 25 was considered abnormal.

Based on the Doppler velocimetry and MBPP results, the study population was divided into four groups:

- A-Normal MBPP and normal Doppler velocimetry

- B-Normal MBPP and abnormal Doppler velocimetry

- C-Abnormal MBPP and normal Doppler velocimetry

- D-Abnormal MBPP and abnormal Doppler velocimetry.

Perinatal outcome was measured in terms of stillbirth/IUD, LBW, Apgar $<7$ at 5 minutes, admission to NICU, neonatal death within 48 hours of delivery, MSL and neonatal seizures within 24-48 hours. Mother and neonate were followed up till they were discharged from the hospital.

\section{Statistical analysis}

Quantitative variables were compared using independent t-test/Mann Whitney test. Qualitative variables were correlated using Chi square test/Fisher exact test. Sensitivity, specificity, NPV, PPV were calculated and pvalue $<0.05$ was considered statistically significant. Data analysis was done using social sciences (SPSS) licensed version 21.0.

\section{RESULTS}

The mean age of the patients was $25.33 \pm 3.48$ years of which majority belonged to the age group 21 to 25 years. Out of these 150 patients, $61(40.67 \%)$ were primigravida and $89(59.33 \%)$ were multigravida. Majority $(41.33 \%)$ were between $37-40$ weeks while $3.33 \%$ were early preterm ( $<34$ weeks) and $25.33 \%$ were late pre-term (3437 weeks). $30 \%$ were post-dated pregnancies. Mean period of gestation was $37.96 \pm 2.32$ weeks. 
Figure 1 shows the women with various high-risk pregnancies that were included in this study.

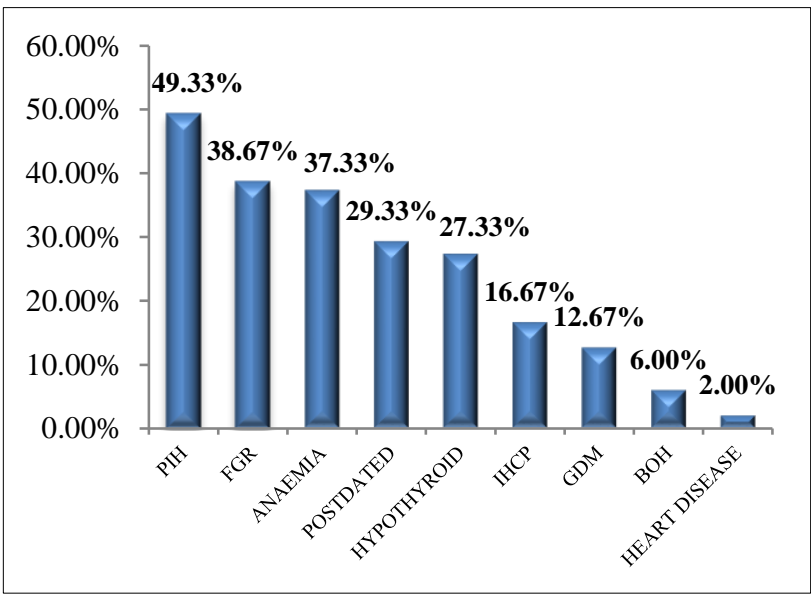

Figure 1: Number of patients with each high-risk factor.

All cases were divided into four groups based on MBPP and Doppler velocimetry. Figure 2 shows the distribution of high-risk pregnancies among the four groups.

A total 28 women went into spontaneous labour and delivered vaginally whereas 83 women required PGE2 gel induction out of which, $59.33 \%$ had vaginal delivery and $40 \%$ had emergency LSCS of which maximum belonged to Group D.

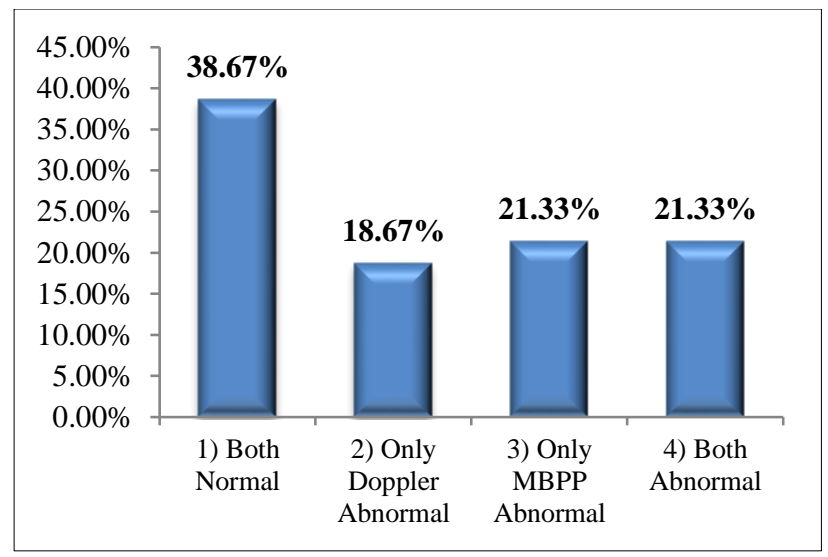

Figure 2: Distribution of high-risk pregnant women in four groups.

\section{Perinatal outcome}

Out of 150 high-risk pregnancies, 95 (63.33\%) had adverse perinatal outcome. Table 1 shows the group-wise details of perinatal outcome.

It was found that highest perinatal complications occurred in Group D with both MBPP and Doppler abnormal, followed by Group $\mathrm{C}$ with only abnormal MBPP and normal Doppler.

This data was statistically significant with $\mathrm{p}$ value $<0.0001$.

Table 1: Perinatal outcome in each group.

\begin{tabular}{|llllll|}
\hline Perinatal outcome & $\begin{array}{l}\text { Group A } \\
\text { (both normal) }\end{array}$ & $\begin{array}{l}\text { Group B (only } \\
\text { Doppler abnormal) }\end{array}$ & $\begin{array}{l}\text { Group C (only } \\
\text { MBPP abnormal) }\end{array}$ & $\begin{array}{l}\text { Group D } \\
\text { (both abnormal) }\end{array}$ & $\begin{array}{l}\text { p value } \\
\text { Liquor }\end{array}$ \\
\hline Nil & - & - & - & - & $2(6.25 \%)$ \\
\hline Blood stained & - & - & - & $1(3.13 \%)$ & - \\
\hline Clear & $58(100 \%)$ & $28(100 \%)$ & $16(50 \%)$ & $22(68.75 \%)$ & - \\
\hline MSL & - & - & $16(50 \%)$ & $7(21.88 \%)$ & $<0.0001$ \\
\hline Low birth weight & $15(25.86 \%)$ & $22(78.57 \%)$ & $14(43.75 \%)$ & $29(90.63 \%)$ & $<0.0001$ \\
\hline Apgar $<7$ & - & $2(7.14 \%)$ & $2(6.25 \%)$ & $9(28.13 \%)$ & $<0.0001$ \\
\hline NICU admission & - & $10(35.71 \%)$ & $12(37.50 \%)$ & $25(78.13 \%)$ & $<0.0001$ \\
\hline Stillbirth & - & - & - & $2(6.25 \%)$ & 0.058 \\
\hline Neonatal seizure & - & - & - & $3(9.38 \%)$ & 0.010 \\
\hline Neonatal death & - & $1(3.57 \%)$ & $1(3.13 \%)$ & $2(6.25 \%)$ & 0.350 \\
\hline $\begin{array}{l}\text { Total with abnormal } \\
\text { perinatal outcome }\end{array}$ & $15(25.86 \%)$ & $22(78.57 \%)$ & $27(84.38 \%)$ & $31(96.88 \%)$ & $<0.0001$ \\
\hline
\end{tabular}

\section{Correlation between MBPP, Doppler and perinatal outcome}

Out of 95 new-borns with adverse perinatal outcome, 58 had abnormal MBPP while only 53 had abnormal
Doppler. Total 23 patients had MSL out of which all the 23 had abnormal MBPP and only 7 of them had abnormal Doppler. MBPP gave correct prediction of immediate perinatal outcome in 107 cases and wrong prediction in 43 cases ( $p$ value < 0.0001) while Doppler gave correct 
prediction of immediate perinatal outcome in 101 cases and wrong prediction in 49 cases ( $\mathrm{p}$ value <0.0001). A total 8 high-risk pregnant women had reversed end diastolic flow (REDF) and 20 had absent end diastolic flow (AEDF) and all these 28 women had abnormal perinatal outcome out of which 17 had abnormal MBPP and 11 had normal MBPP (12/20 AEDF patients had abnormal MBPP and 5/8 REDF patients had abnormal
MBPP). However, this result was not statistically significant ( $\mathrm{p}$ value - 1).

The predictive value of MBPP for adverse perinatal outcome is better than Doppler as shown in Table 2.

Table 2 showing sensitivity, specificity, PPV, NPV and positive likelihood ration of MBPP and Doppler.

Table 2: Sensitivity, specificity, PPV, NPV, positive likelihood ratio.

\begin{tabular}{|llllll|}
\hline & Sensitivity & Specificity & PPV & NPV & Positive likelihood ratio \\
\hline MBPP & $90.62 \%$ & $56.98 \%$ & $61.05 \%$ & $89.09 \%$ & 2.4 \\
\hline Doppler & $88.33 \%$ & $53.33 \%$ & $55.79 \%$ & $87.27 \%$ & 1.89 \\
\hline MBPP + Doppler combined & $96.87 \%$ & $45.76 \%$ & & & \\
\hline Any one abnormal & $86.96 \%$ & $74.14 \%$ & & & \\
\hline
\end{tabular}

\section{DISCUSSION}

This study showed that the highest percentage of perinatal complications occurred in women with both abnormal MBPP and abnormal Doppler (96.88\%). In Group B (only Doppler abnormal) 78.57\% had abnormal perinatal outcome whereas in Group $\mathrm{C}$ (only abnormal MBPP) $84.38 \%$ had abnormal perinatal outcome. Group A where both MBPP and Doppler were normal had the least morbidity similar to study by Padmagirison $\mathrm{R}$ et al. But in their study the number in Group $\mathrm{C}$ was too small for statistical comparison. ${ }^{6}$

In the study by Choudhary $\mathrm{N}$ et al, the highest percentage of perinatal complications, NICU admissions and perinatal deaths were seen in groups with abnormal test results of both NST and velocimetry similar to this study. This study concluded that Doppler velocimetry was better in predicting foetal compromise in comparison to NST in high risk pregnancies. ${ }^{5}$ However, this study had limitations like small sample size, different scans were done by different radiologists resulting in inter-observer variations.

In another study, Gonzalez compared the efficacy of nonstress test, biophysical profile, or abnormal Dopplers for predicting adverse perinatal outcomes in 151 singleton pregnancies with intrauterine growth restriction. Sensitivity, specificity, PPV, NPV of Doppler in predicting adverse perinatal outcomes were $28 \%, 88 \%$, $42 \%, 79 \%$ whereas that of NST were $33 \%, 89 \%, 50 \%$ and $81 \%$ respectively. ${ }^{3}$

In a study comparison of NST, contraction stress test (CST), MBPP and Doppler USG for prediction of foetal acidosis in women with IUGR was done. Similar to this study, they found that the predictive value of NST, CST and MBPP was $57.1 \%$. However predictive value of Doppler velocimetry was only $14.3 \% .^{7}$ A study by
Yelikar et al, maximum neonates from Group C and D had abnormal perinatal outcome like in this study. Their study confirmed that REDF was associated with higher perinatal morbidity. However, the sensitivity of Doppler $(42.1 \%)$ was as good as NST $(42.1 \%)$, while the specificity of NST $(85.9 \%)$ was better than that of Doppler $(65.9 \%){ }^{8}$

In a study determining relationship between Doppler, foetal biophysical profile and foetal acidosis, sensitivity and specificity of NST and BPP was better than umbilical artery S/D ratio. ${ }^{9}$ In another study, sensitivity of MBPP was $60 \%$ and umbilical artery Doppler was $50 \%$ and combination of the two results increased sensitivity to $70 \%$ in predicting perinatal outcome above 36 weeks of gestation. This study concluded that MBPP was more significant than Doppler and their combination was more significant than MBPP alone, similar to this study. ${ }^{10}$

Choudhary $\mathrm{N}$ et al, studied Doppler and MBPP in pregnant women with several other high risk factors other than FGR, like gestational hypertension, diabetes mellitus, post-dated pregnancy. ${ }^{5}$ This study also included high-risk factors other than FGR, like post-dated pregnancy, gestational diabetes mellitus, hypothyroidism, IHCP, anaemia, pregnancy induced hypertension, maternal heart disease and bad obstetric history and found that Doppler or MBPP can predict adverse perinatal outcome even in them. Therefore, both these tests, Doppler and MBPP must be performed in all highrisk pregnant women with or without FGR.

\section{CONCLUSION}

MBPP was proven to be a better predictor of perinatal outcome compared to umbilical artery Doppler ultrasound in high risk pregnant women. MBPP should be done in all high-risk pregnancies even if Doppler is normal. Doppler or MBPP can predict adverse perinatal 
outcome in pregnancy complicated by any high-risk factor irrespective of FGR. Hence, both these antenatal surveillance tests must be performed in all high-risk pregnant women to improve perinatal outcome.

\section{ACKNOWLEDGMENTS}

Authors would like to thank to the institute, VMMC and Safdarjung Hospital, for giving the opportunity to conduct the procedures involved in this study and providing the required equipment for the same.

Funding: No funding sources Conflict of interest: None declared

Ethical approval: The study was approved by the Institutional Ethics Committee

\section{REFERENCES}

1. Kumar MP, Gnanadeep TNV, Dixit UR, Patil PS. Prevalence of high-risk pregnancy in rural Dharwad. IOSR-JDMS. 2015;14:29-32.

2. Shaikh AB, Chidre YV. Comparison of the biophysical profile and modified biophysical profile in prediction of the fetal outcome in pregnancy induced hypertension. Int $\mathrm{J}$ Reprod Contracept Obstet Gynecol. 2017;6:1206-10.

3. Gonzalez JM, Stamilio DM, Ural S, Macones GA, Odibo AO. Relationship between abnormal fetal testing and adverse perinatal outcomes in intrauterine growth restriction. Am J Obstet Gynecol 2007;196:48-51.

4. Arias F, Daftary SN, Bhide AG. eds. Practical guide to high risk pregnancy and delivery. $3^{\text {rd }}$ edition. India. Elsevier Sci Health Sci Div; 2009:17-27.
5. Choudhary N, Sharma BK, Kanungo BK, Yadav R, Rahman H. Assessment of doppler velocimetry versus nonstress test in antepartum surveillance of high risk pregnancy. Int J Reprod Contracept Obstet Gynecol. 2017;6:663-70.

6. Radhika P, Lavanya R. Fetal doppler versus NST as predictors of adverse perinatal outcome in severe preeclampsia and fetal growth restriction. J Obstet Gynecol India. 2006;56:134-8.

7. Shalev E, Zalel Y, Weiner E. A comparison of the non-stress test, oxytocin challenge test, Doppler velocimetry and biophysical profile in predicting umbilical vein $\mathrm{pH}$ in growth retarded fetuses. Int $\mathbf{J}$ Gynecol Obstet. 1993;43:15-9.

8. Sarno AP, Ahn MO, Brar HS, Phelan JP, Platt LD. Intrapartum Doppler velocimetry, amniotic fluid volume, and fetal heart rate as predictors of subsequent fetal distress. I. An initial report. Am J Obstet Gynecol. 1989;161(6 Pt 1):1508-14.

9. Bardakci M, Balci O, Acar A, Colakoglu MC. Comparison of modified biophysical profile and doppler ultrasound in predicting the perinatal outcome at or over 36 weeks of gestation. Gynecol Obstet Invest. 2010;69(4):245-50.

10. Dubiel M, Gudmundsson S, Thuring-Jönsson A, Maesel A, Marsal K. Doppler velocimetry and nonstress test for predicting outcome of pregnancies with decreased fetal movements. Am J Perinatol. 1997;14(3):139-44.

Cite this article as: Malhotra K, Kumari A, Anand HP. Comparison of modified biophysical profile and Doppler ultrasound in prediction of perinatal outcome in high-risk pregnancies. Int J Reprod Contracept Obstet Gynecol 2020;9:2808-12. 